\title{
Womens Sociodemographic and Gyneco-Obstetrical Factors Related to IPTp Observance in Ouidah-Kpomasse-Tori Bossito, Benin
}

\author{
Padonou Sètondji Géraud Roméo, ", Aguemon Badirou ${ }^{1}$, Damien Georgia ${ }^{2}$, \\ Tognifode Mèdessè Véronique ${ }^{3}$, Djossou Elisette ${ }^{1}$, Codjia Estelle ${ }^{4}$, Hinson Antoine Vickey ${ }^{5}$, \\ Ayelo Paul ${ }^{5}$ \\ ${ }^{1}$ Department of Public Health, Faculty of Health Sciences, University of Abomey-Calavi, Cotonou, Republic of Benin \\ ${ }^{2}$ Population and Health Department, Center for Training and Research in Population, University of Abomey-Calavi, Cotonou, Republic of \\ Benin \\ ${ }^{3}$ Department of Obstetric Gynecology, Faculty of Health Sciences, University of Abomey-Calavi, Cotonou, Republic of Benin \\ ${ }^{4}$ Teaching and Research Unit in Community Health and Epidemiology, Faculty of Health Sciences, University of Abomey-Calavi, Cotonou, \\ Republic of Benin \\ ${ }^{5}$ Research and Teaching Unit in Occupational Health and Environment, Faculty of Health Sciences, University of Abomey-Calavi, Cotonou, \\ Republic of Benin
}

\section{Email address:}

geraudpad@yahoo.fr (P. S. G. Roméo)

${ }^{*}$ Corresponding author

\section{To cite this article:}

Padonou Sètondji Géraud Roméo, Aguemon Badirou, Damien Georgia, Tognifode Mèdessè Véronique, Djossou Elisette, Codjia Estelle, Hinson Antoine Vickey, Ayelo Paul. Womens Sociodemographic and Gyneco-Obstetrical Factors Related to IPTp Observance in OuidahKpomasse-Tori Bossito, Benin. Central African Journal of Public Health. Vol. 6, No. 6, 2020, pp. 351-357.

doi: 10.11648/j.cajph.20200606.16

Received: December 1, 2020; Accepted: December 9, 2020; Published: December 25, 2020

\begin{abstract}
Background: In Benin, adherence to intermittent preventive treatment against malaria in pregnant women is below national indicators. This study aimed to determine the rate of IPT1, IPT2, and IPT3 and to investigate the sociodemographic and gyneco-obstetrical factors associated with IPTp's intake. Methods: During a cross-sectional study conducted from October 2017 to February 2018 in southern Benin, 422 women, pregnant in the last trimester or who gave birth less than a month ago were included. Sociodemographic, gynecological and obstetric factors were collected. Logistic regression model was fitted to search for factors associated with IPT's use. Results: The rates of IPT1, IPT2 and IPT3 were $36.49 \%, 26.78 \%$ and $11.14 \%$ respectively. Primiparous mothers $(\mathrm{OR}=1.31$ [1.11-1.92]), women who had attended at least 4 antenatal care visits (ACV) $(\mathrm{OR}=12.93$ [6.27-26.64]) and those who received IPT counseling during their ACV $(\mathrm{OR}=5.27$ [3.02-9.17]) were more likely to take IPT. Conversely, the increase in women's age was associated with a lower probability of taking IPT (OR=0.56 [0.51$0.92])$. No significant association was found with marital status $(\mathrm{p}=0.37)$ and schooling level $(\mathrm{p}=0.38)$. Conclusion: This study confirmed the low use of IPTp for all doses. Our findings suggest strengthening public health interventions to increase women's participation in prenatal consultations by targeting older and multiparous women. Intervention such as organizing intra-community prenatal consultations in addition to those organized at the health center would be useful to improve the national coverage of the IPTp.
\end{abstract}

Keywords: IPT, Parity, Antenatal Care Visit, Woman's Age

\section{Introduction}

In 2016, more than 216 million cases of malaria were reported worldwide, representing an increase of more than 5 million cases compared to 2015 [1]. And Sub-Saharan Africa is the most affected with nearly $90 \%$ of deaths related to malaria $[1,2]$. Every year, more than 25 million of pregnant 
women are at risk of malaria worldwide and especially in Sub-Saharan Africa [1]. Pregnant women represent a particularly vulnerable population. Gestational malaria can be manifested in two ways, namely malaria infection in the circulating peripheral blood and placental malaria infection. The negative consequences of gestational malaria affect both mother and fetus and have been widely described in the literature, including maternal anemia, maternal death, fetal anemia, stillbirth, neonatal death, prematurity and intrauterine growth retardation leading to low birth weight [2-11].

For more than a decade, the World Health Organization (WHO) has recommended intermittent preventive treatment (IPT) for malaria in pregnant women to ensure good protection against this pathology especially in endemic areas like Sub-Saharan Africa. IPT is a fixed combination of 2 molecules, Sulfadoxine-Pyrimethamine (SP). In October 2012, the WHO recommendations were updated [12]: the IPT is administered in supervised dose by a health worker from the 2nd trimester at every antenatal care visit consultation (AVC) until delivery in respecting an interval of at least one month between 2 consecutive doses [12, 13].

Benin, a country located in malaria-endemic area, adopted IPT in 2006 and set the national coverage performance standards at $80 \%$. But after more than 10 years of implementation, and despite the free IPT in pregnant women set up by the national program against malaria, the national coverage of IPT remains low, 40\%. In Ouidah-KpomassèTori Bossito health zone, the IPT coverage is even lower than the national rate and is around 30 to $35 \%$. It therefore seems relevant to investigate the reasons for this low coverage. In general, several parameters can be involved. In this study, we have particularly focused on factors that are directly linked with women such as sociodemographic and gynecoobstetrical factors. Our main objective was to determine the role of these factors on the use of IPTp.

\section{Methods}

\subsection{Study Area Characteristics}

This cross-sectional study conducted during 05 months from October 2017 to February 2018, took place in Ouidah / Kpomassè / Tori-Bossito (OKT) area, located in the Atlantic Department, south of the Republic of Benin. It is a semiurban area of $942 \mathrm{~km}^{2}$, crossed by Lake Ahémé and several satellite streams providing larval breeding, the climate is tropical alternating between two rainy seasons and two dry seasons during the year. In 2016 the population was estimated at 254742 inhabitants (National Institute of Statistics and Economic Analysis, INSAE) and the incidence of malaria was $49.3 \%$ (Ministry of Health, Demographic and Health Survey 2016). The main mosquito vectors that exist in this area are Anopheles gambiae ss and Anopheles funestus. Plasmodium falciparum is the most commonly identified parasite species [14].

\subsection{Study Population}

We included pregnant women in the last trimester or women who gave birth less than a month ago, and permanently living in the study area. Exclusion criteria:

1) Women not eligible to take Sulfadoxine-Pyrimethamine (allergy, taking cotrimoxazole in progress)

2) Refusal to participate

We combined 2 types of sampling methods. The first step was a stratified probability proportional sampling. We have formed 3 strata corresponding to Ouidah, Kpomassè and Tori-Bossito. The size within each stratum was proportional to the size of the population in the corresponding town. The second step was a 3-stage cluster sampling. The first degree was the district of each town, the second degree the village and the third degree the house or the hearth. At each degree a random draw has been made. The size of the sample was calculated using the Schwartz formula: $\mathrm{N}=\varepsilon^{2} \mathrm{pq} / \mathrm{i}^{2}$ with $\varepsilon=1.96$ (for $\alpha=5 \%$ ), $p=$ Prevalence of the IPT in zone $\mathrm{OKT}=35 \%, \mathrm{q}=1-\mathrm{p}=65 \%$, and $\mathrm{i}$ the expected accuracy $=0.05$. We decided to increase the sample size by $20 \%$ to give greater statistical power. The calculated sample size was therefore 420 subjects. A total of 422 women were included in the study.

\subsection{Data Collection}

Date were collected at the health center or at home, during an individual interview where a questionnaire was filled in. Investigators were trained before the start of the field phase and were supervised by a senior epidemiologist.

\subsection{Variables Definition}

Dependent variable

Taking IPT was the dependent variable of our study, qualitative variable, coded 1 when the woman took at least one dose of IPT during pregnancy and coded 0 if no.

Independent variables

1) Sociodemographic factors: age, marital status, level of education, occupation status, ethnic group and religion.

2) Gyneco-obstetrical factors: parity, number of antenatal care visits $(\mathrm{ACV})$, receiving IPT counseling during $\mathrm{ACV}$, pregnancy related hypertension, genital infections and anemia during pregnancy, knowledge of malaria complications on pregnancy.

\subsection{Statistical Analysis}

Inconsistencies, duplicates, and missing data were systematically detected and corrected. After description of the study population, univariate and multivariate analyzes were conducted to investigate factors associated with taking IPTp. We used Chi2 test or Fisher test to compare percentages and Student test to compare means. All variables with $\mathrm{p} \leq 0.20$ in univariate analysis were introduced into a logistic regression model for multivariate analysis. In multivariate, women's schooling status have been recoded in 2 categories: schooled and unschooled women. Statistical 
significance was set at $\mathrm{p}<0.05$. Data were processed using STATA 11® (StataCorp LP, College Station, TX, USA).

\subsection{Ethical}

Women were included on the basis of their free and informed oral consent. Before the start of data collection, public information sessions were organized in the 3 towns by the teams of investigators. Each participant had the right to withdraw from the study at any time.

\section{Results}

\subsection{Use of IPTp}

As shown in figure 1, in the study population, 108 women never took IPT during pregnancy and almost $75 \%$ took at least one dose of IPT. By looking at the different doses of IPT taken by the women, we observed a progressive decrease, $36.49 \%, 26.78 \%$ and $11.14 \%$ respectively for the 1 st dose, the 2 nd dose and the 3 rd dose of IPTp.

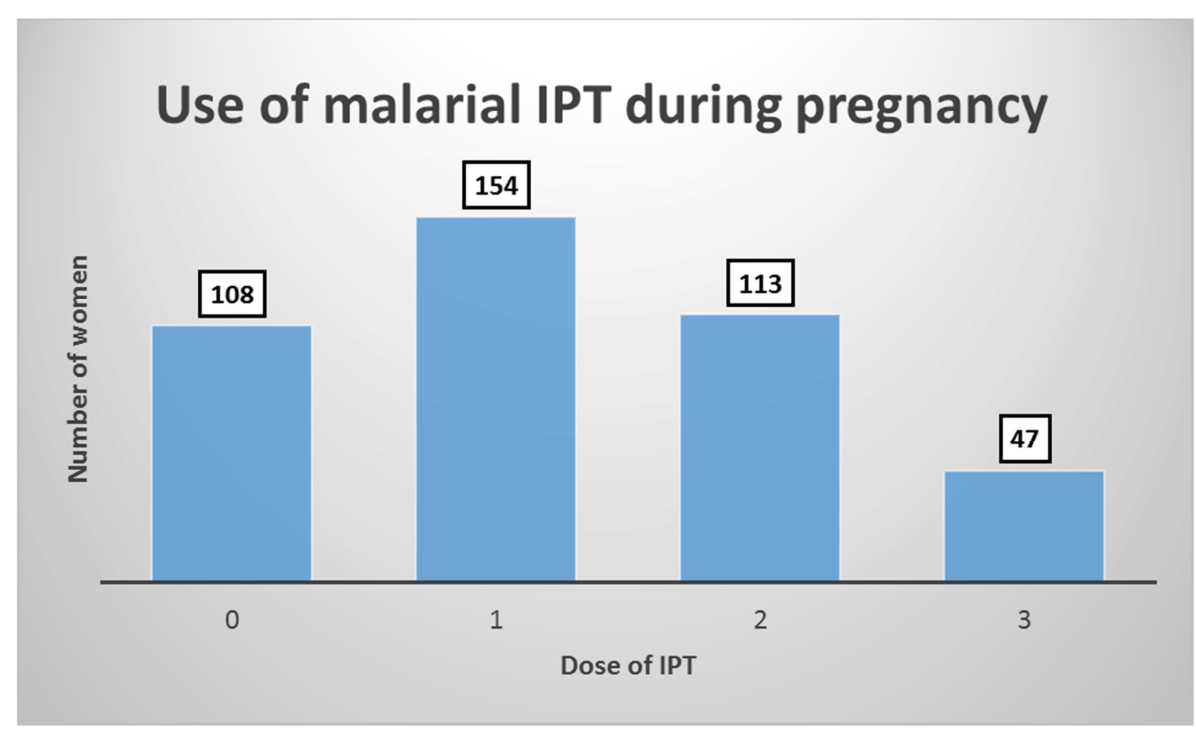

Figure 1. Use of malarial IPTp.

\subsection{Sociodemographic Factors}

Mean age (SD) was 25.63 years $(6.42)$ and the majority of women, $55.21 \%$ were at most 25 years old. Whether in qualitative variable $(p=0.64)$ or quantitative $(p=0.16)$, women's age was not associated with taking IPTp. But our study suggests that women who take the IPTp are younger. Most women were married $(80.09 \%)$, more than half were out of school $(57.35 \%)$. There was no significant difference in using malarial IPTp with women's marital status (0.12) and schooling status (0.19). Majority of participants were artisan, $45.97 \%$ or merchants, $39.10 \%$. Six percent were students. The dominant ethnic group and religion were respectively Fon (83.65\%) and Christian (60.90\%). We have not found any significant association between these sociodemographic factors and the use of malarial IPTp. (Table 1)

Table 1. Sociodemographics factors.

\begin{tabular}{|c|c|c|c|c|}
\hline & \multirow{2}{*}{ N (\%) or Mean (SD) } & \multicolumn{2}{|c|}{ Use of malarial IPTp (N or Mean) } & \multirow{2}{*}{$P$} \\
\hline & & Yes & No & \\
\hline Age & $25.63(6.42)$ & 24.88 & 25.89 & 0.16 \\
\hline Age in class & & & & 0.64 \\
\hline$\leq 25$ & $233(55.21)$ & 170 & 63 & \\
\hline ] 25-35] & $154(36.49)$ & 116 & 38 & \\
\hline$>35$ & $35(8.29)$ & 28 & 7 & \\
\hline Marital status & & & & 0.12 \\
\hline Married & $338(80.09)$ & 250 & 88 & \\
\hline Not married & $84(19.91)$ & 64 & 20 & \\
\hline Schooling status & & & & $0.19^{\mathrm{a}}$ \\
\hline Unschooled & $242(57.35)$ & 176 & 66 & \\
\hline Primary and secondary & $175(41.47)$ & 134 & 41 & \\
\hline higher & $5(1.18)$ & 4 & 1 & \\
\hline Occupation & & & & $0.43^{\mathrm{a}}$ \\
\hline Unemployed & $23(5.45)$ & 15 & 8 & \\
\hline Student & $27(6.40)$ & 20 & 7 & \\
\hline Artisan & 194 (45.97) & 141 & 53 & \\
\hline
\end{tabular}




\begin{tabular}{|c|c|c|c|c|}
\hline & \multirow{2}{*}{ N (\%) or Mean (SD) } & \multicolumn{2}{|c|}{ Use of malarial IPTp ( $\mathrm{N}$ or Mean) } & \multirow{2}{*}{$P$} \\
\hline & & Yes & No & \\
\hline Merchant & $165(39.10)$ & 126 & 39 & \\
\hline Civil servant & $13(3.08)$ & 12 & 1 & \\
\hline Ethnic group & & & & $0.68^{\mathrm{a}}$ \\
\hline Fon & $353(83.65)$ & 257 & 96 & \\
\hline Tori & $29(6.87)$ & 26 & 3 & \\
\hline Mina & $40(9.48)$ & 31 & 9 & \\
\hline Religion & & & & 0.65 \\
\hline Christian & $257(60.90)$ & 184 & 73 & \\
\hline Muslim & $39(9.24)$ & 29 & 10 & \\
\hline Traditional & $126(29.86)$ & 101 & 25 & \\
\hline
\end{tabular}

${ }^{a}$ Fisher's test

\subsection{Gynecological and Obstetrical Factors}

The majority of women were primiparous, $56.64 \%$. Parity was significantly related to taking IPTp; indeed, there were more primiparous mothers among women who took IPTp. Mean number (SD) of antenatal care visits (ACV) was 3.49 (2.26). Women who complied with IPTp had a significantly higher number of ACV than others $(p=0.000)$. This association was observed even when the number of ACV was analyzed as qualitative variable $(<4 ; \geq 4)$. Nearly $60 \%$ of women reported receiving IPTp counseling during their ACV and this was significantly positively associated with taking IPTp ( $\mathrm{p}=0.000)$. Among women surveyed, $13.98 \%$ had anemia, $22.04 \%$ had a genital infection, and $7 \%$ had a pregnancy-related hypertension. Almost 35\% of women reported they did not know the complications of malaria on pregnancy. We found that pregnancy-related hypertension and genital infections during pregnancy were significantly related to taking IPTp. (Table 2).

Table 2. Gyneco-obstetrical factors.

\begin{tabular}{|c|c|c|c|c|}
\hline & \multirow{2}{*}{ N (\%) or Mean (SD) } & \multicolumn{2}{|c|}{ Use of malarial IPTp ( $N$ or Mean) } & \multirow{2}{*}{$\mathbf{P}$} \\
\hline & & Yes & No & \\
\hline Parity & & & & 0.01 \\
\hline Primiparous & $239(56.64)$ & 174 & 65 & \\
\hline Multiparous & $183(43.36)$ & 140 & 43 & \\
\hline Number of ACV & $3.49(2.26)$ & 4.21 & 1.42 & 0.000 \\
\hline Number of ACV (in category) & & & & 0.000 \\
\hline$<4$ & $233(55.21)$ & 135 & 98 & \\
\hline$\geq 4$ & $189(44.79)$ & 179 & 10 & \\
\hline IPT counseling during ACV & & & & 0.000 \\
\hline Yes & $248(58.77)$ & 216 & 32 & \\
\hline No & $174(41.23)$ & 98 & 76 & \\
\hline Anemia during pregnancy & & & & 0.72 \\
\hline Yes & $59(13.98)$ & 45 & 14 & \\
\hline No & $363(86.02)$ & 269 & 94 & \\
\hline Genital infections during pregnancy & & & & 0.004 \\
\hline Yes & $93(22.04)$ & 80 & 13 & \\
\hline No & $329(77.96)$ & 234 & 95 & \\
\hline Pregnancy related hypertension & & & & $0.005^{\mathrm{a}}$ \\
\hline Yes & $29(6.87)$ & 28 & 1 & \\
\hline No & $393(93.13)$ & 286 & 107 & \\
\hline Knowledge of malaria complications on pregnancy & & & & 0.79 \\
\hline Yes & $278(65.88)$ & 208 & 70 & \\
\hline No & $144(34.12)$ & 106 & 38 & \\
\hline
\end{tabular}

${ }^{\text {a }}$ Fisher's test

\subsection{Factors Associated with Taking IPTp. Multivariate Analysis}

The variables: age, parity, number of ACV, counseling on IPTp during ACV were significantly associated with taking IPTp. Increasing age, the probability of taking the IPTp decreases. Older women have poor adherence to using malarial IPTp compared to younger $(\mathrm{OR}=0.56, \mathrm{p}=0.031)$. The probability of taking IPTp was higher in primiparous women than in multiparous $(\mathrm{OR}=1.31, \mathrm{p}=0.04)$ and in women who had attended at least $4 \mathrm{ACV}$ compared to those who had less than 4 ACV $(\mathrm{OR}=12.93, \mathrm{p}=0.000)$. The occurrence of genital infections during pregnancy seemed to increase the probability of taking IPTp but this effect was 
borderline $(\mathrm{P}=0.09)$. Pregnancy-related hypertension was no longer associated with the use of IPTp in multivariate

analysis. (Table 3).

Table 3. Factors associated with use of malarial IPTp. Multivariate analysis by logistic regression.

\begin{tabular}{lll}
\hline \multirow{2}{*}{ Variables } & Use of malarial IPTp & \\
\cline { 2 - 3 } & Adjusted OR [95\% CI] & $\boldsymbol{p}$ \\
\hline Age & $0.56[0.51 ; 0.92]$ & 0.031 \\
Marital status (Married) & $1.74[0.81 ; 2.34]$ & 0.37 \\
Schooling status (Schooled) & $1.49[0.78 ; 2.09]$ & 0.38 \\
Parity (Primiparous) & $1.31[1.11 ; 1.92]$ & 0.04 \\
Number of ACV $\geq 4$ & $12.93[6.27 ; 26.64]$ & 0.000 \\
IPT counseling during ACV (Yes) & $5.27[3.02 ; 9.17]$ & 0.000 \\
Genital infections during pregnancy & $1.87[0.89 ; 3.92]$ & 0.09 \\
Pregnancy related Hypertension & $1.29[0.67 ; 2.87]$ & 0.51 \\
\hline
\end{tabular}

\section{Discussion}

This study, which concerned 422 women in the OuidahKpomassè-Tori Bossito area, aimed to determine the compliance with the recommended doses of IPTp and to determine women's sociodemographic and gynecoobstetrical associated factors.

Just over $25 \%$ of women never took IPT during pregnancy. The rates were $36.49 \%, 26.78 \%$ and $11.14 \%$ respectively for IPT1, IPT2 and IPT3 doses. Primiparous, women who had followed up at least 4 antenatal care visits, and women who received IPTp counseling during their ACV had a significantly greater probability of taking IPTp. In contrast, age was a reducing factor, older women had a lower probability of taking IPTp.

The rate noted was quite low and gradually decreased for the 2nd and 3rd doses of IPT. Benin's national performance targets for IPT were not met. The low prevalence in the OKT health zone is consistent with what is observed in other departments and at national level in Benin. IPT coverage during pregnancy is low and requires a lot of effort to be made to reach the set goals. According to the latest WHO Malaria Report 2017, the coverage rate of IPT3 was $19 \%$ on average in 23 African countries [1], higher than the $11.14 \%$ observed in our work. In Africa, the IPTp uptake rate varies depending on country. In Senegal, a rate similar to ours was observed with $80.35 \%$ of women taking at least one dose of IPTp [15]. In a recent study in Ghana, observed prevalence was higher than ours: $98.5 \%$ of women received at least one dose of IPTp and 71\% received fully 3 doses of IPTp [16]. Lower rates were found in rural Tanzania where $16 \%$ of women surveyed never took IPTp during pregnancy, 54\% took one dose and $34 \% 2$ doses [17].

Primiparous mothers more complied with taking IPTp compared to multiparous, our observations are shared by other authors [18-22]. This is because primiparous mothers use health center services more often, such as antenatal care and even postnatal care, as several studies have shown [23, 24], since IPTp doses are given and supervised by health workers during these consultations. The same observation was also made in Nigeria [25]. Several reasons could explain this fact. These primiparous mothers are mostly young. They have little experience in maternity, are often worried about the progress of pregnancy and need to be reassured by the advices of health workers and regular medical examinations during antenatal consultations. On the other hand, older women have more children and are more confident because they have a lot of experience from previous pregnancies. Therefore they go to health center only in case of proven illness. Such a situation involves risks on mother-and-child health and it is necessary to revise this by adopting a multisectoral approach. This approach must take into account the improvement of the health system and the performance of health centers, must better include both pregnant women and their husbands.

Women who attended at least $4 \mathrm{ACV}$ and those who received IPTg counseling during ACV have a higher probability of taking IPTp. This result is consistent since, according to WHO recommendations, IPTp should be taken directly and supervised by a health worker during an antenatal consultation. Our results are consistent with what is widely described in LMICs, highlighting the important positive role that antenatal consultation has played in taking IPTp and in the outcome of pregnancy in general $[15,16,18,19,21,22,26$, 27]. The antenatal consultation allows a good monitoring of the pregnancy and a better follow-up of the fetal intrauterine growth. It can early detect any abnormality that may occur during pregnancy. In our study population, less than half of women, $44.79 \%$, attended at least $4 \mathrm{ACV}$ as recommended by WHO. In LMICs this recommendation is poorly respected with rates ranging from $30 \%$ to $40 \%$ of women having attended at least $4 \mathrm{ACV}$ [28]. The almost $45 \%$ observed in our study are higher than the national rate of $29.4 \%$ of women having had at least $4 \mathrm{ACV}$ according to the statistics of the Ministry of Public Health in 2016, and represent a better gyneco-obstetrical follow-up of the pregnancy. However, one of the measures proposed in this work, the advanced strategy of ACV in homes and households, will improve this important indicator of maternal and child health. Organizing intracommunity prenatal consultations in addition to those organized at the health center would be useful to improve the national coverage of the IPTp.

During the prenatal consultations, apart from the medical examination, advice is given to women on the progress of the pregnancy, the precautionary measures to be taken, 
information is given on the pathologies related to pregnancy and the first signs of emergency. This advice includes malaria and malaria prophylaxis with IPT. In our study, we found that these advices were significantly associated with taking IPTp. Studies in Ghana, Kenya, and Malawi also found that women positively valued the advice given by health workers during prenatal consultations [29-31].

We observed in our study during univariate analysis, that women who had genital infections during pregnancy and those who presented pregnancy related hypertension, were more likely to receive IPTp. On the medical level, there is no direct link between genital infections during pregnancy, pregnancy related hypertension and taking IPTp. But the relationship is rather indirect. These variables, which can be described as instrumental variables, actually reflect the woman's presence at the health center outside the normal prenatal consultation schedule. When the pregnant woman is healthy and has no signs of worry, she does not feel the need to come to the health center. She prefers to go about her business, to devote herself to her professional and incomegenerating activities. But when she is ill she goes to the health center for examination and proper care. And it is during this consultation that the health staff by stripping the woman's health book realizes that this dose of IPTp has not been taken. The woman is then given the required dose of IPTp in addition to other care related to the pathology that brought her to the health center. Our results shed light on a sociological aspect of people's perception of health and care. In their logic, when we are not sick, we do not have to go to the health center. And we only go when the state of health requires it. This implies proposals for actions with an advanced strategy where health workers and community relays go inside the community, homes and households to offer ACV services and distribute IPTp free of charge. As in the case of vaccination campaigns, for example, children who do not come to the health center are vaccinated at home by health workers who move from household to household. This type of public health intervention already exists in Benin for other health issues and should be extended to the ACV. Positive results have been observed in other countries [32].

In populations living in rural or semi-rural areas, the level of education was described as a determining factor in observance of health care in general. Women with a higher level of education are more receptive to awareness and education messages, health informations. They better understand the risks associated with various diseases and more often adopt the recommended preventive measures. In our study population, the variable "schooling status" was not associated with the use of malarial IPTp. In Benin, like many other African countries, information, education and communication messages, communication messages for behavior change are explained and disseminated in the local languages of target populations during outreach sessions. This promotes their understanding and their adherence to messages. This may explain in our study the lack of link between women's level of education and the use of malarial IPTp.

In this work, we have chosen to focus only on factors related to pregnant women. But the compliance with recommendations on IPTp can also depends on other factors such as the sufficient presence and availability of qualified health worker, the supply, free distribution and efficient management of the Sulfadoxine-Pyrimethamine stock by health authorities in the OKT zone. It is therefore relevant to carry out additional studies taking into account in particular, the management of Sulfadoxine-Pyrimethamine stock made by the health centers, as well as other parameters such as perception of health by the pregnant woman, index of household wealth.

\section{Conclusion}

Our results showed that it is older and multiparous women who do not adhere to the schedule for intermittent preventive malaria treatment. This can be corrected by increasing the number of prenatal consultations through awareness raising, information, education and by organizing intra-community prenatal consultations.

\section{Declaration of Interest}

The authors declare that they have no competing interests.

\section{Acknowledgements}

We are grateful for all women who participate in this study. We thank the entire staff of field investigators.

\section{References}

[1] WHO, World Malaria Report 2017.

[2] WHO, World Malaria Report 2013.

[3] Bouyou-Akotet, M. K., et al., Anaemia and severe malarial anaemia burden in febrile Gabonese children: a nine-year health facility based survey. J Infect Dev Ctries, 2013. 7 (12): p. 983-9.

[4] De Beaudrap, P., et al., Impact of malaria during pregnancy on pregnancy outcomes in a Ugandan prospective cohort with intensive malaria screening and prompt treatment. Malar J, 2013. 12: p. 139.

[5] Desai, M., et al., Epidemiology and burden of malaria in pregnancy. Lancet Infect Dis, 2007.7 (2): p. 93-104.

[6] Eisele, T. P., et al., Malaria prevention in pregnancy, birthweight, and neonatal mortality: a meta-analysis of 32 national cross-sectional datasets in Africa. Lancet Infect Dis, 2012. 12 (12): p. 942-9.

[7] Hill, J., et al., Factors affecting the delivery, access, and use of interventions to prevent malaria in pregnancy in sub-Saharan Africa: a systematic review and meta-analysis. PLoS Med, 2013. 10 (7): p. e1001488.

[8] Kendjo, E., et al., Mortality patterns and site heterogeneity of severe malaria in African children. PLoS One, 2013. 8 (3): p. e58686. 
[9] Sohail, M., et al., Prevalence of Malaria Infection and Risk Factors Associated with Anaemia among Pregnant Women in Semiurban Community of Hazaribag, Jharkhand, India. Biomed Res Int, 2015. 2015: p. 740512.

[10] Takem, E. N. and U. D'Alessandro, Malaria in pregnancy. Mediterr J Hematol Infect Dis, 2013. 5 (1): p. e2013010.

[11] WHO, World Malaria Report. 2011.

[12] WHO, Updated WHO Policy Recommendation. 2012. Oct, Intermittent Preventive Treatment of malaria in pregnancy using Sulfadoxine Pyrimethamine (IPTp SP). 2012.

[13] WHO, Policy brief for the implementation of intermittent preventive treatment of malaria in pregnancy. World Health Organization. 2014 (1: 12).

[14] Corbel, V., et al., Multiple insecticide resistance mechanisms in Anopheles gambiae and Culex quinquefasciatus from Benin, West Africa. Acta Trop, 2007. 101 (3): p. 207-16.

[15] Mbengue, M. A. S., et al., Factors influencing the use of malaria prevention strategies by women in Senegal: a crosssectional study. Malar J, 2017. 16 (1): p. 470.

[16] Ibrahim, H., et al., Factors influencing uptake of intermittent preventive treatment of malaria in pregnancy using sulphadoxine pyrimethamine in Sunyani Municipality, Ghana. Pan Afr Med J, 2017. 28: p. 122.

[17] Ayubu, M. B. and W. B. Kidima, Monitoring Compliance and Acceptability of Intermittent Preventive Treatment of Malaria Using Sulfadoxine Pyrimethamine after Ten Years of Implementation in Tanzania. Malar Res Treat, 2017. 2017: p. 9761289.

[18] Amoran, O. E., A. A. Ariba, and C. A. Iyaniwura, Determinants of intermittent preventive treatment of malaria during pregnancy (IPTp) utilization in a rural town in Western Nigeria. Reprod Health, 2012. 9: p. 12.

[19] Exavery, A., et al., Factors affecting uptake of optimal doses of sulphadoxine-pyrimethamine for intermittent preventive treatment of malaria in pregnancy in six districts of Tanzania. Malar J, 2014. 13: p. 22.

[20] Kibusi, S. M., E. Kimunai, and C. S. Hines, Predictors for uptake of intermittent preventive treatment of malaria in pregnancy (IPTp) in Tanzania. BMC Public Health, 2015. 15: p. 540 .

[21] Nwaefuna, E. K., et al., Effectiveness of Intermittent
Preventive Treatment in Pregnancy with SulphadoxinePyrimethamine against Submicroscopic falciparum Malaria in Central Region, Ghana. J Parasitol Res, 2015. 2015: p. 959427.

[22] Orish, V. N., et al., Prevalence of intermittent preventive treatment with sulphadoxine-pyrimethamine (IPTp-SP) use during pregnancy and other associated factors in SekondiTakoradi, Ghana. Afr Health Sci, 2015. 15 (4): p. 1087-96.

[23] Sahle, G., Ethiopic maternal care data mining: discovering the factors that affect postnatal care visit in Ethiopia. Health Inf Sci Syst, 2016. 4: p. 4.

[24] Tarekegn, S., L. S. Lieberman, and V. Giedraitis, Determinants of maternal health service utilization in Ethiopia: analysis of the 2011 Ethiopian Demographic and Health Survey. BMC Pregnancy Childbirth, 2014. 14: p. 161.

[25] Akinyemi, J. O., R. F. Afolabi, and O. A. Awolude, Patterns and determinants of dropout from maternity care continuum in Nigeria. BMC Pregnancy Childbirth, 2016. 16 (1): p. 282.

[26] Azizi, S. C., et al., Uptake of intermittent preventive treatment for malaria during pregnancy with SulphadoxinePyrimethamine (IPTp-SP) among postpartum women in Zomba District, Malawi: a cross-sectional study. BMC Pregnancy Childbirth, 2018. 18 (1): p. 108.

[27] Bouyou-Akotet, M. K., D. P. Mawili-Mboumba, and M. Kombila, Antenatal care visit attendance, intermittent preventive treatment and bed net use during pregnancy in Gabon. BMC Pregnancy Childbirth, 2013. 13: p. 52.

[28] UNICEF, The State of the World's Children 2016. UNICEF Global Databases. NEW YORK. 2016.

[29] Menaca, A., et al., Local illness concepts and their relevance for the prevention and control of malaria during pregnancy in Ghana, Kenya and Malawi: findings from a comparative qualitative study. Malar J, 2013. 12: p. 257.

[30] Pell, C., et al., Prevention and management of malaria during pregnancy: findings from a comparative qualitative study in Ghana, Kenya and Malawi. Malar J, 2013. 12: p. 427.

[31] Pell, C., et al., Factors affecting antenatal care attendance: results from qualitative studies in Ghana, Kenya and Malawi. PLoS One, 2013.8 (1): p. e53747.

[32] Namazzi, G., et al., Working with community health workers to improve maternal and newborn health outcomes: implementation and scale-up lessons from eastern Uganda. Glob Health Action, 2017. 10 (sup4): p. 1345495. 